\title{
Study on the Acoustic Characteristics of Sunwapta Falls
}

\author{
Zhixing Tian', Bong-Young Kim² and Myung-Jin Bae ${ }^{3 *}$ \\ ${ }^{1}$ Soong-sil University, Department of Information and telecommunication Engineering, Seoul, 06978, Korea. \\ Orcid Id : 0000-0003-3882-2459 \\ ${ }^{2}$ Soong-sil University, Department of Information and telecommunication Engineering, Seoul, 06978, Korea. \\ Orcid Id : 0000-0002-3553-039X \\ ${ }^{3}$ Soong-sil University, Department of Information and telecommunication Engineering, Seoul, 06978, Korea. \\ Orcid Id : 0000-0002-7585-0400
}

\begin{abstract}
The auditory system, as a human alert system, has an important connection with human emotions, and both psychologically and physically affected by the sound heard. Therefore, people's health is closely related to the health of their sound environment. If exposed to big city noise for a long time, this may damage your health. But in the natural environment, most white noise and pink noise have the effect of relieving stress and relaxing the body and mind, which is beneficial to health. The Sunwapta Fall sound studied in this article is also one of the healthy sounds. Use spectrum analysis to get its acoustic characteristics which is similar to white noise and has three special formants. Then, in order to study its psychoacoustic characteristics, brainwave measurement experiment. As a result, Sunwapta Fall sound stimulated more alpha and beta brainwaves. This also proves that it can really reduce stress and contribute to health.
\end{abstract}

Keyword: Sunwapta Falls, white noise, acoustic characteristics, health, stress

\section{INTRODUCTION}

With the development of society, although the medical level and living standard have been improved, a lot of people are still in a sub-health state, which is not only associated with people in a high-load working environment, greater social pressure and unhealthy lifestyle, and has a great connection with the environmental pollution around us. Noise pollution is also one of the reasons that damage people's health. The sound environment is closely related to people's health. People's hearing system is a keen warning system, and it works even when sleeping. If a certain sound appears or disappears out of the ordinary, it will cause the auditory system to alert and activate the brain. Make the brain produce a stress response, and further psychological and physiological adjustments to make action responses. Most Studies have stated that sound can affect people's emotional changes. For example, People can feel relaxed when listening to soothing songs, and excited when listening to rock songs. The direct impact of sound on human emotions, Witch mean sound can have a specific psychological effect on people, thereby affecting people mental health. This psychological effect is directly related to the acoustic characteristics of sound. Urban noise is mainly distributed in low-frequency high-energy noise. Prolonged exposure to this sound environment can reduce people's cognition and behavior, make them tired, and trigger negative emotions such as irritability, anger, and depression. Therefore, it is very important to improve people's sound environment. For people living in the city, on weekends, there are some good way to maintain health, which is away from the city for mountain climbing, walking on the beach or forest, outdoor camping and other outdoor activities close to nature [1] [2].

Natural environmental sounds are mostly white noise and pink noise that are comfortable and relaxing. For example, the sound of wind, rain, flowing stream, waterfall, etc. Being in such a sound environment can make people feel relaxed and beneficial to them health. This paper is a specific study of Sunwapta Falls, one of the most famous waterfalls in Canada. Sunwapta Falls is one of the many waterfalls in the Rocky Mountains. The water flow first bypasses a small island and divides into two branches and finally merges into a strong current. And then pour down from the cliff. Although the drop is not large, no more than 18 meters, but due to the huge amount of water and the confluence of the two streams, the scenery is quite spectacular. And because of its terrain, it has unique acoustic characteristics [3] [4].

This paper is a study of the acoustic and psychoacoustic characteristics of Sunwapta Falls. Mainly the frequency spectrum analysis and brain wave analysis of Sunwapta Falls sound. In chapter 2, we introduced the characteristics and applications of white noise. In chapter 3 , we conducted power spectrum analysis and energy spectrum analysis. In chapter 4 , introduced brain waves and measured and compared city sound and Sunwapta Falls brain wave maps. In chapter 5, conclusion.

\section{WHITE NOISE}

Acoustically, the energy of white noise is evenly distributed in all frequency bands, including all sound frequencies in human ear's audible range $(20 \sim 20000 \mathrm{~Hz})$. White noise has a flat spectrum, that is, the power spectrum energy is the same in different frequency bands, so white noise is a smooth straight line parallel to the frequency axis in the linear spectrum. It exists widely in natural environments, such as the sound of rain, 
sea waves, wind blowing leaves, waterfalls, etc. In human lives, the sound of air conditioning, fan sound, and the sound of TV and radio when there is no signal are similar to white noise [5] [6].

White noise is generally considered to be a natural and comfortable sound because it has a flat power spectral density over a wide frequency spectrum. In physiology and psychology, many studies have stated that white noise can bring positive effects to people. White noise is also often used as a means of sound therapy. At present, it is mainly used to treat tinnitus, help sleep, and cure the mental concentration disorder of some hyperactive children. Because its energy in the entire frequency band is the same, it can shield the noise accumulated by energy and keep the surrounding environment relatively quiet. Using this principle, many kinds of white noise generator has been developed, and placing it in the office can increase people's attention and make work efficiently [7].

However, excessive exposure to white noise will have the opposite effect, will reduce the health of the nervous system and impair cognitive ability. Prolonged exposure to loud white noise increases the risk of depression. So, most studies have stated that listening to white noise properly can relax and improve attention, but once it exceeds a certain limit, it will have an adverse effect on the brain. And natural white noise is more soothing than artificial white noise [8] [9].

\section{SPECTRUM ANALYSIS}

In the time domain, white noise is found to be a continuous random sound signal with consistent sound energy. There are no specific rule and rhythms. From the spectrogram, it can be seen that the energy is more evenly distributed in the frequency range of $20 \mathrm{~Hz}$ to $10 \mathrm{KHz}$, and there is no excessive concentration of energy distribution. It is shown in Figure 1.

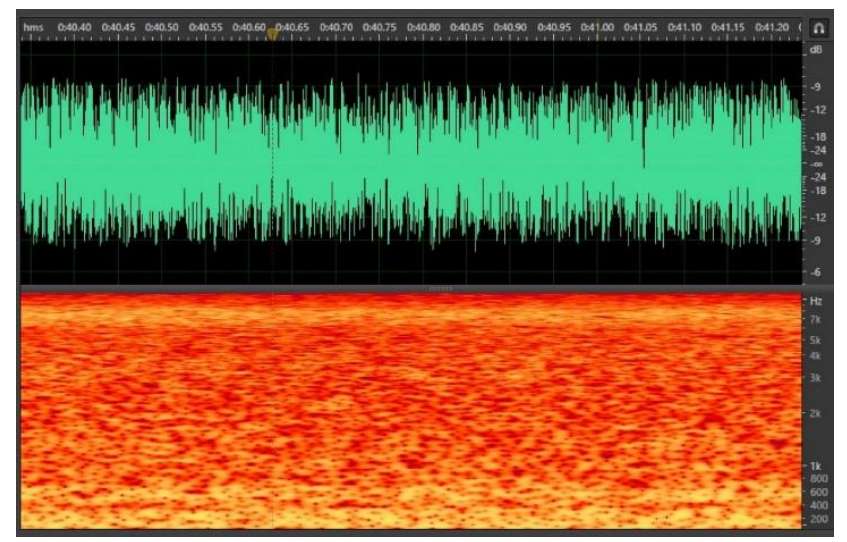

Figure 1. Waveform and spectrogram
The spectrum analysis of Sunwapta Falls revealed its unique acoustic characteristics. As shown in Figure 2, the overall power spectral density is relatively flat in the frequency range of $20 \mathrm{~Hz} \sim 11 \mathrm{KHz}$, and the spectral characteristics are similar to white noise, indicating that it also has some characteristics of white noise. In the range of $100 \sim 300 \mathrm{~Hz}, 300 \sim 700 \mathrm{~Hz}$ and $6 \sim$ $9 \mathrm{KHz}$, three formant structures appear. This is closely related to the water flow, terrain, and height difference of Sunwapta Falls. The first formant $(100 \sim 300 \mathrm{~Hz})$ is formed due to the resonance of the water flow sound and the surrounding geographical space environment in this vast space. The second formant $(300 \sim 700 \mathrm{~Hz})$ is because two streams of water converge to form a turbulent stream, and the sound frequency of turbulent flow is higher, which forms a higher frequency formant with the surrounding geographical space environment. The third formant $(6 \sim 9 \mathrm{KHz})$ is the high-frequency acoustic resonance generated when the water flow falls from a height and beats the rock and the water surface. Studies of forest sounds in autumn and winter have shown that similar highfrequency formants appear when people step on fallen leaves or snow. This high-frequency formant energy can induce $\beta$ waves in the human brain. So that it can be used as a treatment for people with autism [11] [12].

The acoustic characteristics of Sunwapta Falls include not only the acoustic characteristics of white noise, but also the different characteristics brought by the three formants. This constitutes the unique acoustic characteristics of Sunwapta Falls. Because sounds with different acoustic characteristics can bring people different psychological and physiological effects, listening to the sound of Sunwapta Falls will have a unique soothing experience. White noise can induce alpha brain waves, which makes people quickly enter a quiet and relaxed state. At the same time, beta brain waves induced by the formant structure of the high frequency band will improve people's attention and cognitive ability. It will make people enter a quiet and relaxed state of meditation with high concentration and cognitive ability, which is very suitable for thinking [13] [14].

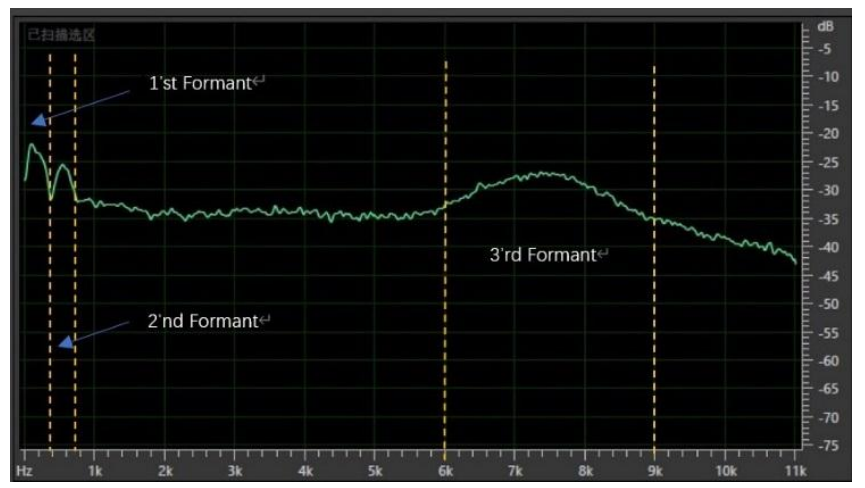

Figure 2. Spectrum analysis of Sunwapta waterfall sound 


\section{BRAIN WAVE MEASUREMENT}

\subsection{Definition of brain waves}

EEG signals reflect the activity of cerebral cortical neurons. Observation of EEG is an important means to obtain information about brain activity and an objective indicator to reflect the state of brain function. The EEG study found that, according to the frequency and amplitude characteristics of EEG signals, it is mainly divided into four types of EEG waves: $\delta$ wave, $\theta$ wave, $\alpha$ wave, and $\beta$ wave. Beta waves are usually divided into low beta waves $(\beta \mathrm{L})$ and high beta waves $(\beta \mathrm{H})$. These brain waves correspond to different frequency domain ranges. In order, the frequency of $\delta$ wave, $\theta$ wave, $\alpha$ wave, $\beta \mathrm{L}$ wave, $\beta \mathrm{H}$ wave gradually increases, and the amplitude gradually decreases. The appearance of different brain waves reflects different brain functional activities [15] [16].

$\delta$ wave, frequency $1-3 \mathrm{~Hz}$, amplitude $20-200 \mu \mathrm{V}$, mostly appear in deep sleep state, neurons in cerebral cortex are in a state of simultaneous relaxation, and do not participate in the process wave of information processing. $\theta$ wave, frequency $4-7 \mathrm{~Hz}$, amplitude $100-150 \mu \mathrm{V}$, it is a brain wave that appears between awake and sleep. And it is related to the memory process. It also occurs when emotionally stimulated, especially when disappointed or frustrated. $\alpha$ wave, frequency $8-13 \mathrm{~Hz}$, amplitude $20-100 \mu \mathrm{V}$, is the main waveform recorded in normal quiet state, sober and closed eyes, indicating that in quiet state, the cerebral cortical nerve cells are in a state of relaxation and preparation for activity. The appearance of the $\alpha$ wave can quickly make people feel relaxed, so many people relax by listening to the alpha wave music. The $\beta$ wave frequency is $14-$ $30 \mathrm{~Hz}$, and the amplitude is $5-20 \mu \mathrm{V}$. It appears when people realize behaviors and actions. Usually, the brain thinks about problems or conducts intelligence. The wave will be converted into this wave during cognitive activities. The $\beta$ wave reflects the alert state of the human brain. The nerve cells of the cerebral cortex are in a state of intense activity, which is the excitement of the cerebral cortex. The continuous appearance of a large number of $\beta$ waves will give people a lot of pressure and tension.

\subsection{Brain wave measure experiment and results}

The experiment first recorded the traffic environment sound and Sunwapta Falls sound for one minute each. The brainwave test site is in a quiet laboratory, and the test subject is a 22-yearold male college student. The test instrument is a two-channel brain wave measuring instrument with a sampling rate of 512 $\mathrm{Hz}$ and a bandwidth of $45 \mathrm{~Hz}$ produced by Neuro Harmory $\mathrm{S}$. After setting the measuring instrument for the test object, the test object remains closed eyes and quiet. The sound is played for three minutes, and its brain wave is measured, and then the operation is repeated after 5 minutes of rest. Finally, the brain wave measurement of the two sound environments is completed. Figure 3 is an EEG Isopotential Mapping of the Sunwapta waterfall sounds, and Figure 4 is an EEG Isopotential Mapping of urban traffic noise.

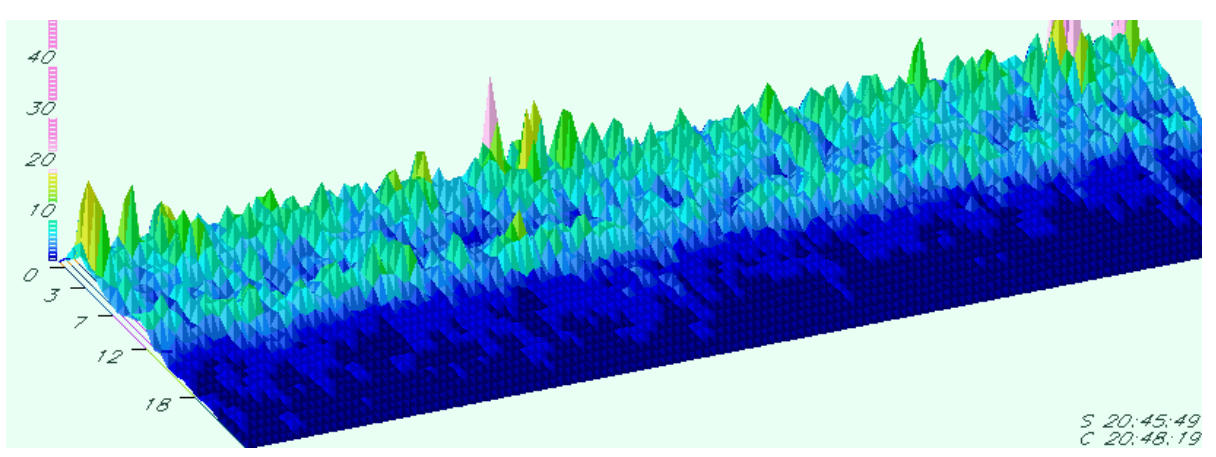

Figure 3. EEG Isopotential Mapping of the Sunwapta waterfall sounds

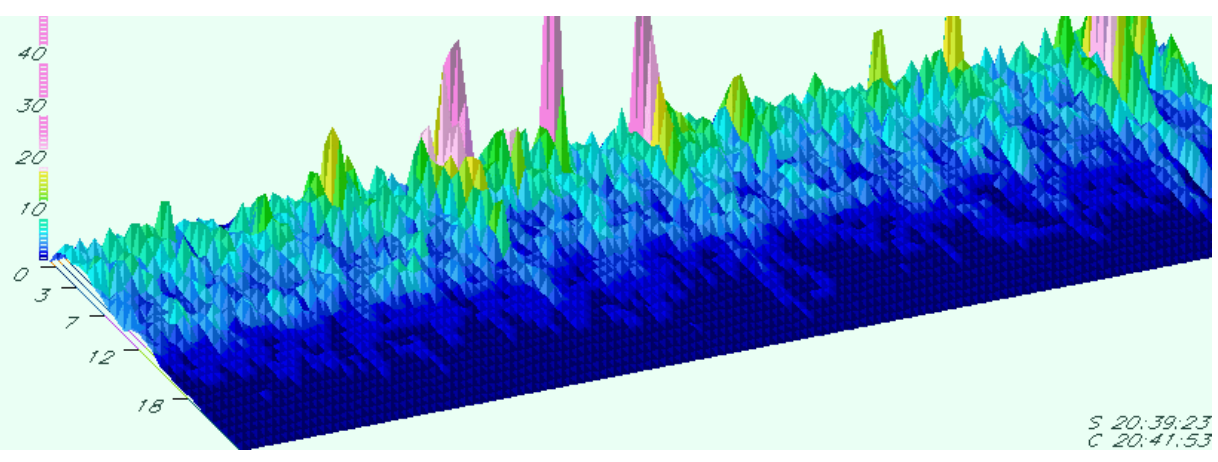

Figure 4. EEG Isopotential Mapping of urban traffic noise 
As can be seen from the electroencephalogram, the waterfall sound reduces the $\theta$ wave, and more $\alpha$ waves and $\beta$ waves appear. This means that waterfall sounds can excite $\alpha$ and $\beta$ waves in our brain and suppress $\theta$ waves. The $\alpha$ wave makes people's brains enter a quiet state of relaxation. At the same time, because the $\theta$ wave decreases and the $\beta$ wave increases, so that the brain remains awake and highly focused. At this time, people will feel relaxed and have a clear mind. This is a very comfortable state and has a positive effect on people's health. On the contrary, the sound of urban traffic makes the brain appear more $\theta$ waves, and inhibits the generation of $\alpha$ waves, and $\beta$ waves are slightly reduced. There are many studies have stated that more $\theta$ waves and less $\beta$ waves can make people feel tired and have less concentration. Such a brain wave pattern for a long time is easy to induce depression. This also shows that different sounds have different effects on people's brain wave activity. Therefore, for people who have been in the urban traffic noise for a long time, listening to the sound of waterfalls can get the effect of Relieve stress and concentration. Table 1 shows the voltage values of various brain waves of two sounds. Figure 5 is Figure 5 is a comparison of various brain waves of two sounds [17] [18].

Table 1. Voltage values of various brain waves of two sounds

\begin{tabular}{|c|l|l|l|l|l|}
\hline $\begin{array}{c}\text { Brain wave( }(\boldsymbol{V}) \\
\text { Sound type }\end{array}$ & $\boldsymbol{\delta}$ & $\boldsymbol{\Theta}$ & $\boldsymbol{\alpha}$ & $\boldsymbol{\beta L}$ & $\boldsymbol{\beta H}$ \\
\hline $\begin{array}{c}\text { Sunwapta } \\
\text { Falls sound }\end{array}$ & 10.83 & 7.50 & 5.98 & 2.06 & 0.72 \\
\hline Traffic noise & 10.73 & 9.08 & 4.31 & 1.77 & 0.63 \\
\hline
\end{tabular}

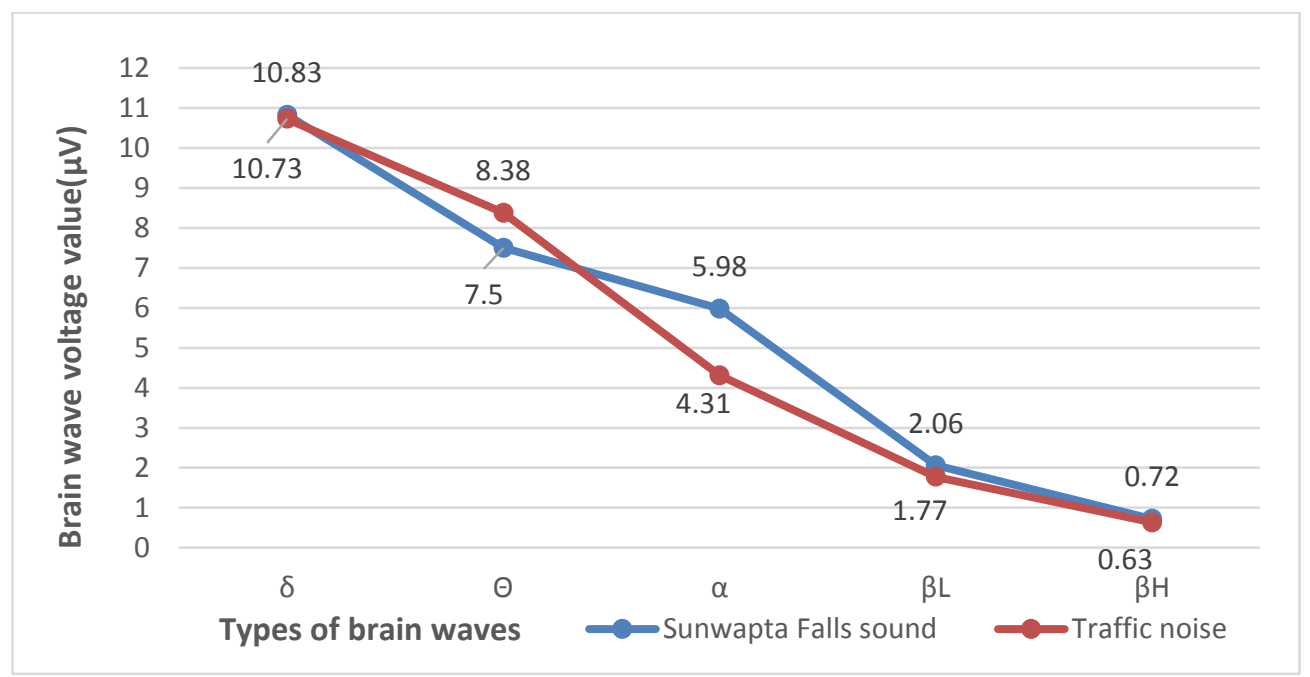

Figure 5. Comparison of various brain waves of two sounds

\section{CONCLUSION}

The sound environment is closely related to people's health. Especially people living in cities are deeply troubled by noise pollution, which damages their physical and mental health. In fact, there are various healthy sounds in nature. They can not only make people feel comfortable and relaxed, but also keep people healthy. For example, the sound of wind, rain, waves, waterfalls, and running water in nature. Listening to these sounds can relieve people's stress and relax.

The Sunwapta fall sound studied in this paper is also a sound that is beneficial to health. Because of its unique terrain, it constitutes its unique acoustic characteristics. In the spectrum analysis, it is found that its frequency density curve is flat, which is similar to white noise. It also has three resonance formants. So, these acoustic characteristics also make
Sunwapta fall sound have unique psychoacoustic characteristics. White noise can excite alpha brain waves, and formants appearing in high frequency bands can excite beta brain waves. In the brain wave test experiment, it is also proved that listening to the sound of Sunwapta waterfall will excite more $\alpha$ and $\beta$ brain waves and suppress $\theta$ brain waves. People will feel relaxed and focused, and their bodies will be full of vitality.

Therefore, for people who have been exposed to urban noise for a long time, stay away from the noisy city and get close to nature. Listening to the sound of nature can improve the sound environment. In this healthy acoustic environment, it can reduce people's stress, increase attention, and benefit health. And mountain climbing, hiking, camping and other activities are healthy sports. It is good for our physical and mental health that join these activities. 


\section{REFERENCE}

[1] Loewen, Laura J.; Peter Suedfeld (1992-05-01). "Cognitive and Arousal Effects of Masking Office Noise". Environment and Behavior. 24 (3): 381-395. doi:10.1177/0013916592243006.

[2] Kim, B.-Y \& Bae, M.-J. (2018). "A study on the sound characteristics analysis of drum-sound rock". Journal of Engineering and Applied Sciences. 13. 4414-4418. 10.3923 .

[3] Domantė Kučikienè1, and Rūta Praninskiene "The impact of music on the bioelectrical oscillations of the brain". Acta Med Litu. 2018; 25(2): 101-106\.

[4] Baker, Mary Anne; Dennis H. Holding (July 1993). "The effects of noise and speech on cognitive task performance". Journal of General Psychology. 120 (3): 339-355. doi:10.1080/00221309.1993.9711152. ISSN 0022-1309. PMID 8138798.

[5] Rausch, V. H. (2014). White noise improves learning by modulating activity in dopaminergic midbrain regions and right superior temporal sulcus. Journal of cognitive neuroscience, 1469-1480.

[6] Ahn IS., Kim BY., You KB., Bae MJ. (2019) “A Study on the Characteristics of an EEG Based on a Singing Bowl's Sound Frequency". In: Lee R. (eds) Software Engineering Research, Management and Applications. SERA 2018. Studies in Computational Intelligence, vol 789. Springer, Cham.

[7] Wu D (2018) Hearing the Sound in the Brain: Influences of Different EEG References. Front. Neurosci. 12:148. doi: 10.3389/fnins.2018.00148.

[8] López, HH; Bracha, AS; Bracha, HS (September 2002). "Evidence based complementary intervention for insomnia". Hawaii Med J. 61 (9): 192, 213. PMID 12422383

[9] Yi, E.-Y \& Bae, M.-J. (2015). "Research on modeling of sound on mountain summits by atmospheric pressure difference". 18. 4413-4419.

[10] Bong-Young Kim, Zhixing Tian, Myung-Jin Bae. "A study on the causes of revenge psychology of Klaxon sound". International Journal of Engineering Research and Technology Volume 12, Issue 12, 2019, Pages 29472952.

[11] Soderlund, Goran; Sverker Sikstrom; Jan Loftesnes; Edmund Sonuga Barke (2010). "The effects of background white noise on memory performance in inattentive school children". Behavioral and Brain Functions. 6 (1): 55. doi:10.1186/1744-9081-6-55. PMC 2955636. PMID 20920224.

[12] GAes, Leonardo. (2018). Binaural beats: Brain wave induction and the use of binaural beats to induce brain wave patterns. Current Research: Integrative Medicine. 03. 10.4172/2529-797X.1000030.

[13] Seonggeon Bae, Myungin Bae. "A study on the effects of depression using the weighting white noise". Acoustical Society of America, Volume 139, Issue 4, 10.1121/1.4949855.

[14] Kim, B.-Y \& Yi, E.-Y \& Bae, M.-J. (2019). "A study on the distinguish of the defective product of ceramic toilet by sound characteristics". Journal of Engineering and Applied Sciences. 14. 1247-1252. 10.3923.

[15] H Yeo, Kyoung-Su; Kim, Myung-Sook; Bae, MyungJin." Acoustic Characteristics of the Forest Sounds Inducing Sleep". International Information Institute (Tokyo). Information; Koganei Vol. 18, Iss. 10, (Oct 2015): 4407-4412.

[16] Sammler D, Grigutsch M, Fritz T, Koelsch S. Music and emotion: electrophysiological correlates of the processing of pleasant and unpleasant music. Psychophysiology. 2007. March; 44(2): 293-304.

[17] Ray WJ, Cole HW. "EEG alpha activity reflects attention demands and beta activity reflects emotional and cognitive processes". Science. 1985. May 10; 228(4700): 750-2.

[18] Lee E-J, Bhattacharya J, Sohn C, Verres R. "Monochord sounds and progressive muscle relaxation reduce anxiety and improve relaxation during chemotherapy: a pilot EEG study". Complement Ther Med. 2012. December; 20(6): 409-16. 